\title{
TREATMENT OF RECURRENT ORAL APHTHOUS ULCERATIONS
}

Krassanai W.. Treatment of recurrent oral aphthous ulcerations. Annal Dent Univ Malaya 1999; 6: 58 - 59.

\section{INTRODUCTION}

Recurring aphthous ulceration is one of the commonest conditions affecting the mucous membrane of the mouth. It causes pain and suffering to the patient, more or less on an intermittent basis. The real aetiology is not definite. Although trauma maybe a precipitating cause, the hormonal, immunological and the psychosomatic aspect have been suggested as underlining causes. From the diverse observations, it has been proposed that there are a variety of causes or some unifying factor, which maybe contributing to some of the causes of oral aphthous ulcerations. From the author's own experience, as an aphthous sufferer, he proposes that spicy-hot food and the change in climate may play some part.

Clinical manifestation of aphthous ulcer maybe grouped as minor aphthous (Miculicz type), herpetiform and major aphthous.Behcet's syndrome is characterized by aphthous stomatitis associated with eye lesions and genital ulceration.

The most important feature, for diagnosis of aphthous ulcerations, is the history of recurrence at more or less regular intervals. It should be differentiated from herpectic stomatitis and oral cancer.

\section{Management of Aphthous Ulcerations \\ Systemic and Psychological Investigation}

There is a need to investigate whether there are underlining systemic causes that might play a part in the development of this disease. Malnutrition, consumption of high calorie food, constipation and deficiency in some particular elements and vitamins e.g. iron, folic acid and vitamin $\mathrm{B} 12$ should be investigated and treated.

The patient who has had a history of depression and psychological disturbances or is under stressful conditions of life should seek help and be treated parallel with the local treatment. The patient should be advised against any eating habits that may precipitate or aggravate the condition for example eating fried food regularly, consuming too much very spicy-hot food and not drinking enough water.

\section{Local Management}

The patient should have good oral hygiene, in order to facilitate the healing and the reduction of pain, from local irritation and secondary infection. Local management includes:

\section{$2.1 \quad$ Tetracycline}

The use of tetracycline, $250 \mathrm{mg}$ in $10 \mathrm{ml}$ of fluid, held in the mouth for two or three minutes, three times a day.

\subsection{Chlorhexidine}

A $0.2 \%$ solution of chlorhexidine can be used as a mouth wash, three times daily after meals and held in the mouth for at least one minute.
Krassanai Wangrangsimakul

Dental Faculty,

Prince of Songkhla University,

Haadyai, Songkhla, 90112, THAILAND

\subsection{Corticosteroids}

Corlan (hydrocortisone hemisuccinate $2.5 \mathrm{mg}$ ), pellets to be allowed to dissolve in the mouth, three times a day. Triamcinolone in orabase is to be applied to the lesion, three times a day, after meals and before going to bed.

Figure 1 - Asiatic Pennywort (Centella Asiatica)

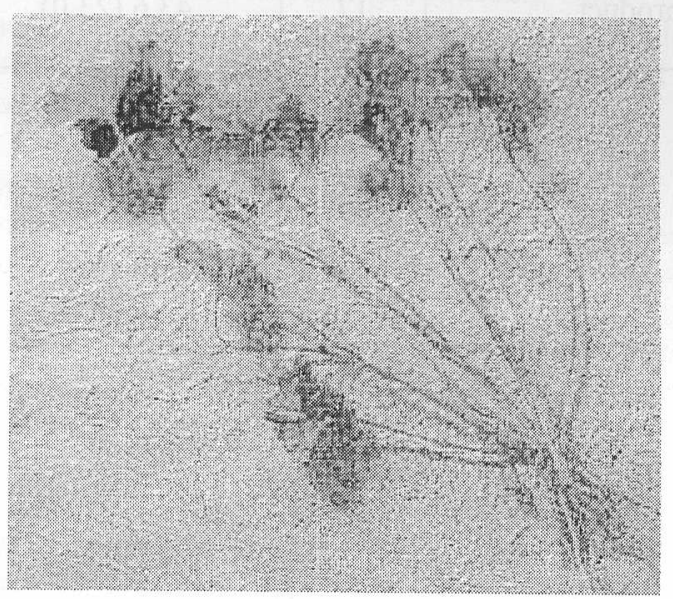

Traditional Medicinal Herbs as an Alternative Medicine Chinese and Thai people have long known and treated aphthous ulcers as "ulcers of the inner heat", with a medicinal herb called Asiatic Pennywort (Centella asiatica). It has been used as a drink not only to satisfy the thirst but also to reduce the "inner heat" so that it helps healing of the aphthous ulcers.

Centella asiatica has long been used as a traditional medicine, for ulcers and wounds, in Europe and Asia. The chemical component from the extracts of Asiatic pennywort is of the terpenic compounds i.e., asiatica acid, methyasiatate, madecassic acid, asiaticoside and madecassoside.

There is still not enough evidence on the pharmacology of Centella asiatica. There is a study on the use of Centella asiatica oral mucoadhesive gel, for treating aphthous ulcerations, by Associate Professor Suchada Prasertwittayakarn and Associate Professor Chaiyo Chaicharntippayut, of the Faculty of Pharmacy, Chulalongkorn University, Bangkok.

They investigated the clinical efficacy for treating aphthous ulcers, by using Centella asiatica gels, with 0.50 , 1.00 and $2.00 \%$ extract in C934P base comparing with 
Table 1 - Mean cure rate of aphthous ulcers and mean in-vivo adhesion time of $0.5,1.0,2.0 \% \mathrm{w} / \mathrm{w}$ Centella asiatica gels, placebo and a commercial triamcinolone acetonide (TA) product

\begin{tabular}{|l|c|c|}
\hline \multicolumn{1}{|c|}{ Treatment } & $\begin{array}{c}\text { No. of } \\
\text { patients }\end{array}$ & $\begin{array}{c}\text { Mean cure rate(SD) } \\
\text { Mm/d }\end{array}$ \\
\hline Centella gel 0.5\% & 17 & $0.690(0.398)$ \\
Centella gel 1.0\% & 19 & $0.859(0.455)$ \\
Centella gel 2.0\% & 16 & $0.959(0.387)$ \\
Placebo & 18 & $0.287(0.259)$ \\
TA product & 17 & $0.837(0.305$ \\
& & \\
\hline \multicolumn{1}{|c|}{ Treatment } & No. of & Mean in-vivo \\
& patients & adhesion time \\
\hline Centella gel 0.5\% & 17 & $37.3(23.3)$ \\
Centella gel 1.0\% & 19 & $36.6(18.3)$ \\
Centella gel 2.0\% & 16 & $30.1(9.7)$ \\
Placebo & 18 & $32.8(16.9)$ \\
TA product & 17 & $43.6(23.0)$ \\
\hline
\end{tabular}

placebo and triamcinolone acetonide orabase in 87 subjects. It was found that $0.50,1$ and $2 \%$ Centella asiatica gels and triamcinolone acetonide showed indifferent decreasing rate of lesion size, but significantly higher rates in comparison with placebo $(\mathrm{P}<0.05)$ Additionally, all treatments showed no significant difference in retention times in 69 subjects (Table1).

\section{REFERENCE}

1. Kosalwatna S, Shaipanich C, Phanganda K. The effect of one percent Centella asiatica cream on chronic ulcers. Siriraj Hosp. 1988; (6): 456-460

2. Singh B, Rastogi RP. An investigation of the triterpenses of Centella asiatica. Phytochemistry 1969; 8: 917-921.

3. Bonte F, Dumas M, Chaudagne C, Meybeck A. Influence of Asiatic acid, Madecassic acid, and Asiaticoside on human collagen 1 systhesis. Planta Med. 1994; 60: 133-135.

4. Harris D, Robinson..JR. Drug delivery via the mucous membranes of the oral cavity. J. Pharm. Sci. 1992; 81 (1): $1-10$. 